\title{
Empleo e impuestos a la nómina en Colombia. Un análisis de los efectos ocupacionales de la Ley 1607 de 2012 de Reforma Tributaria ${ }^{1}$
}

\author{
STEFANO FARNÉ ${ }^{2}$ \\ DAVID ARTURO RODRÍGUEZ ${ }^{3}$
}

\section{Resumen}

En su esencia, la ley de Reforma Tributaria en Colombia reduce en 13,5 puntos porcentuales las contribuciones que gravan la nómina a cargo de las empresas, ingresos que, en buena medida, el fisco recupera a través de impuestos a las personas naturales para las cuales aumenta la carga tributaria directa. Con esta reforma, el gobierno espera generar más empleos -entre 400 mil y 1 millón- y lograr una mejor distribución del ingreso -el índice de Gini pasaría de 57,3 a 55,4 . Este documento emplea los argumentos de un simple modelo de equilibrio parcial para estudiar los efectos sobre el mercado de trabajo derivados de modificaciones en los impuestos a la nómina y reseña una serie de recientes trabajos empíricos internacionales en ese sentido. Aporta unos cálculos alternativos del impacto ocupacional esperado de la reforma tributaria en Colombia. Asimismo avanza algunas consideraciones acerca de su probable efecto sobre la distribución del ingreso. Nuestras conclusiones ponen en entredicho los optimistas pronósticos del gobierno colombiano tanto en materia de empleo como de distribución del ingreso.

Palabras clave: Ley 1607 de 2012, Reforma Tributaria, Índice de Gini, Impuesto para la Equidad -CREE-, Impuestos a la nómina, Generación de empleo, Parafiscalidad.

\section{Introducción}

El día 26 de diciembre de 2012, el presidente de la República sancionó la Ley 1607, de Reforma Tributaria. De acuerdo con la exposición de motivos que en su momento presentó el gobierno nacional, esta ley persigue dos principales objetivos: generar más empleo y lograr una mejor distribución del ingreso.

${ }^{1}$ Los autores agradecen a los profesores José Antonio Ocampo -Columbia University, de Nueva York- y Jhon James Mora -Universidad Icesi, de Cali- y a los doctores Hugo López -gerente del Banco de la República de Medellín- y Ricardo Rocha -director de Consultoría Económica y Empresarial de Bogotá- por sus valiosos comentarios y sugerencias. Las opiniones, errores u omisiones aquí contenidos son responsabilidad exclusiva de los autores.

${ }^{2}$ M.Sc. in Economics, Universidad de Londres, Inglaterra. Director Observatorio del Mercado de Trabajo y Seguridad Social, Universidad Externado de Colombia. Correo-e: dirobservatorio@ uexternado.edu.co.

${ }^{3}$ M.Sc. in Economics, Universidad de Southampton, Inglaterra. Investigador Observatorio del Mercado de Trabajo y Seguridad Social, Universidad Externado de Colombia. Correo-e: david. rodriguez@uexternado.edu.co. 
Fundamentalmente, el primer objetivo se conseguiría con una reducción de 13,5 puntos porcentuales de unos impuestos parafiscales a cargo de las empresas, es decir, con una disminución de cerca del $8 \%$ de los costos laborales totales ${ }^{4}$; el segundo con una reducción de la retención a la fuente para niveles bajos de ingresos, el recorte de las deducciones tributarias permitidas y un incremento progresivo de las tarifas de impuesto a la renta, todo enmarcado en un nuevo Impuesto Mínimo Alternativo Nacional -IMAN- que se introduce con la reforma y que fija, a partir de los 3.460 .000 pesos mensuales, unos umbrales mínimos de contribuciones que van creciendo con el nivel de renta ${ }^{5}$.

En cuanto a empleos, el gobierno nacional estima generar entre 400 mil y un millón de nuevos puestos de trabajo formales en los próximos dos años; con respecto a la distribución del ingreso, espera reducir 1,9 puntos del índice de desigualdad de Gini, que pasaría de 57,3 a 55,4 (República de Colombia, 2012: 63).
Este documento discute los efectos sobre el mercado de trabajo provocados por modificaciones en los impuestos a la nómina ${ }^{6}$ en un contexto de equilibrio parcial y reseña una serie de recientes trabajos empíricos internacionales cuyas conclusiones ponen en discusión los optimistas pronósticos del gobierno colombiano en materia de empleo. Al mismo tiempo aporta unos cálculos alternativos del impacto ocupacional esperado de la reforma tributaria en Colombia. También avanza algunas consideraciones acerca de los probables efectos de la reforma sobre la distribución del ingreso.

Consta de siete secciones de las cuales esta introducción es la primera. Las cuatro secciones sucesivas abordan los temas relacionados con tributación y generación de empleo, así como se ha indicado en el acápite anterior. La sección 6 hace una sucinta referencia a la distribución del ingreso. Finalmente, una última sección recoge los principales argumentos presentados a lo largo del texto y trae algunas lecciones de política.

\footnotetext{
${ }^{4}$ Los 13,5 puntos de parafiscalidad gravan solo el salario básico, de manera tal que representan un $8 \%$ de los costos laborales totales, salariales y extrasalariales (ver Cárdenas, 2012).

${ }^{5}$ Formalmente, las empresas siguen siendo responsables de la financiación del Instituto Colombiano de Bienestar Familiar -antes de la reforma destinaban el 3\% de su nómina salarial para tal fin-, del Servicio Nacional de Aprendizaje (2\%, antes de la reforma) y de la salud de sus trabajadores $-8,5 \%$, antes de la reforma-. Para garantizar esta financiación se crea un nuevo impuesto sobre la renta denominado "para la equidad" CREE de $8 \%$ (9\% durante los primeros tres años) de destinación específica. Al mismo tiempo, sin embargo, la tarifa del impuesto general a la renta para las personas jurídicas baja en 8 puntos, de 33\% a 25\%. Las empresas dejan de pagar 13.5 puntos porcentuales de aportes parafiscales que terminan cobrándose en buena parte a las personas naturales para las cuales aumenta la carga tributaria directa. La reducción de la parafiscalidad no aplica a empresas no contribuyentes del impuesto a la renta-del sector público, fundaciones, ONG, por ejemplo-y para los asalariados de empresas contribuyentes con ingresos mensuales superiores a diez salarios mínimos. También son excluidas de este beneficio las sociedades declaradas como zonas francas a 31 de diciembre de 2012, comprendidas las que a esa fecha radicaron su solicitud. Finalmente, vale la pena resaltar que, en la práctica, la reforma tributaria provoca efectos sobre la carga impositiva, tanto de las personas naturales como de las empresas, más complejos de los aquí reseñados de manera simple y resumida. Tener en cuenta esta complejidad no modificaría ni los lineamientos de nuestro análisis, ni las conclusiones alcanzadas.

${ }^{6}$ Los términos parafiscalidad, impuestos al trabajo e impuestos a la nómina son aquí utilizados indiferentemente como sinónimos.
} 
2. Impuesto al trabajo y empleo: algunas consideraciones teóricas

En el marco del modelo básico de equilibrio parcial del mercado laboral empleado por la literatura económica tradicional, a continuación mostramos los efectos que pueden darse sobre el empleo y los salarios como consecuencia de la introducción o de modificaciones en los impuestos que gravan al factor trabajo.

En el modelo básico, un mercado de trabajo perfectamente competitivo y sin impuestos a la nómina se encuentra en equilibrio cuando demanda $\mathrm{D}_{\mathrm{o}}$ y oferta $\mathrm{S}_{\mathrm{o}}$ se igualan. Esta situación se representa en el Gráfico 1, donde el salario de equilibrio se ubica en $\mathrm{w}_{\mathrm{o}}$ y el nivel de ocupación, en $\mathrm{E}_{\mathrm{o}}$.

La introducción de un gravamen a la nómina provoca un desplazamiento de la curva de demanda hacia el interior en el monto del impuesto tax ${ }_{1}$. La nueva curva de demanda de trabajo $\mathrm{D}_{1}$ representa el salario que ahora recibe el trabajador $\left(\mathrm{w}_{1, \mathrm{tra}}\right)$. Mientras que la anterior curva de demanda representa el costo laboral total por trabajador -salario más impuesto-que ahora sostienen las empresas $\left(\mathrm{w}_{1, \mathrm{em}}\right)$.

Es evidente que, respecto al salario inicial $\mathrm{w}_{\mathrm{o}}$, los valores de $\mathrm{w}_{1, \text { tra }} \mathrm{y} \mathrm{w}_{1, \text { em }}$ implican que el impuesto tax ${ }_{1}$ termina siendo compartido por trabajadores y empleadores. A través de costos laborales más altos por trabajador -en una magnitud igual a la línea clara- y de salarios más bajos por trabajador -línea oscura-. Este efecto de salarios más bajos debido al impuesto es conocido en la literatura económica como desplazamiento del impuesto hacia los salarios -efecto pass through-, es decir que los trabajadores comparten la carga del impuesto con sus salarios.

Supongamos que tax ${ }_{1}$ sea equivalente a la parafiscalidad que actualmente grava la nómina de las empresas colombianas y que la Ley 1607 de reforma tributaria implique una reducción al nivel de tax ${ }_{2}$. En este caso la curva de demanda se desplazaría desde $\mathrm{D}_{1}$ hasta $\mathrm{D}_{2}$.

\section{Gráfico 1}

Efectos de los parafiscales sobre el nivel de salarios y empleo

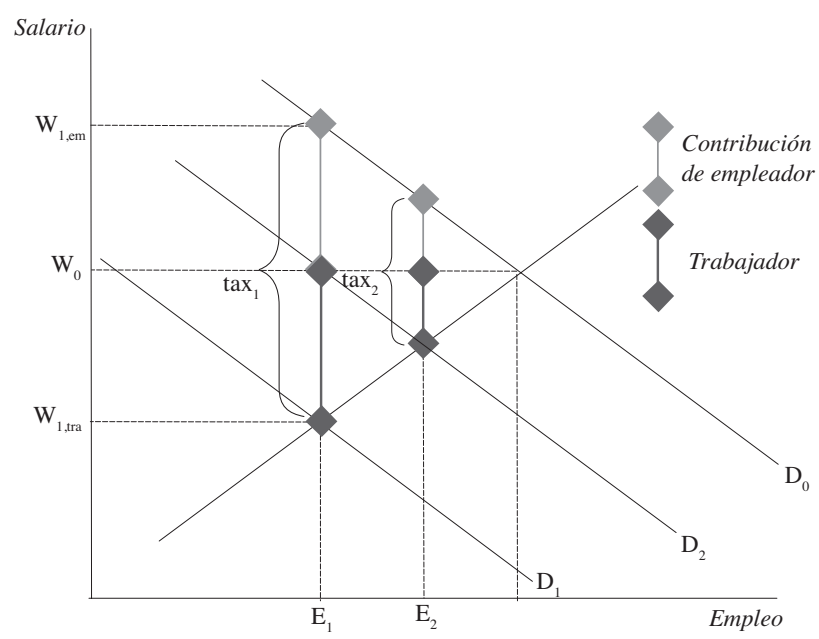


Como consecuencia, en el marco de nuestro modelo, los costos laborales totales caerían pero no en la misma cuantía en la que cayó el impuesto debido a que parte de la reducción del tributo se transforma en mayores salarios para los trabajadores, es decir el efecto pass through opera en dirección opuesta debido a que en esta ocasión se redujeron los gravámenes ${ }^{7,8}$.

En contra de la creencia común de que la legislación determina quién paga estas contribuciones o quién se beneficia con su desmonte, en un mercado laboral perfectamente competitivo son las elasticidades de las curvas de oferta y demanda de trabajo ${ }^{9}$ las que determinan las participaciones que deben asumir unos u otros. En especial, entre más insensible sea la oferta de trabajo ante cambios en el salario o más sensible sea la demanda de trabajo al salario, una mayor proporción del pago del impuesto es transferido a los trabajadores vía menores salarios, es decir, hay un mayor pass through, y en caso de una reducción del impuesto una mayor parte de esta reducción es transferida a los trabajadores vía mayores salarios.

Ahora bien, de lo anterior se deriva que si el pass through es elevado, los efectos sobre el empleo de una reforma tributaria resultan de menores proporciones, dado que gran parte de los aumentos -disminuciones- de impuestos se transforman en menores -mayores-salarios y no logran afectar de manera significativa la demanda de trabajo de las empresas.

En general, si bien el impacto sobre el nivel de ocupación de la introducción o modificación de un impuesto al factor trabajo es distinto dependiendo de si el mercado de trabajo es perfectamente competitivo o no, podemos decir que depende principalmente de cinco factores, a saber:

a) Elasticidad de la demanda de trabajo a los costos laborales;

b) Elasticidad de la oferta de trabajo a los costos laborales;

$\mathrm{Y}$, adicionalmente, de:

c) La valoración de los trabajadores de los parafiscales. Este punto a tener en cuenta para completar el análisis de impuestos al trabajo es aclarado por Summers (1989), quien afirma que es fundamental la valoración que hacen los trabajadores de los servicios recibidos con el impuesto, ya que determina en qué monto están dispuestos a ver reducido su salario con tal de tener acceso a ellos;

d) La existencia de un salario mínimo como restricción activa de los empleadores. Dado que, al mismo tiempo, el Estado fija el salario mínimo y el factor de impuestos, a las empresas les resulta imposible transferir a menores salarios la imposición de gravámenes que afectan la nómina compuesta por trabajadores de salario mínimo. En el caso de una disminución de los impuestos al trabajo, la restricción no es taxativa, pero el salario

\footnotetext{
${ }^{7}$ Nuevamente, en el Gráfico 1 la línea clara representa los costos laborales por trabajador que asume el empleador (nótese que estos han disminuido con respecto al escenario sin reforma tributaria) y la línea oscura el menor salario percibido por los trabajadores (nótese que el salario ha aumentado con respecto al escenario sin reforma tributaria).

${ }^{8}$ Igualmente el nivel de empleo con la reforma aumenta relativo al escenario sin ella, hasta ubicarse en $\mathrm{E}_{2}$.

${ }^{9}$ El concepto de elasticidad demanda (oferta) de trabajo al salario se refiere a cuanto cambia porcentualmente la oferta (demanda) de trabajadores ante una aumento en un $1 \%$ en el salario.
} 
mínimo puede operar como un referente de los pagos de la mano de obra poco calificada.

e) El poder de negociación de los trabajadores que ante reducciones o alzas en los gravámenes a la nómina presionan para obtener mayores salarios o menores reducciones de éste, respectivamente.

Ceteris paribus, en el caso que nos interesa de una disminución de los impuestos al trabajo, el efecto sobre el empleo es mayor si la elasticidad de la demanda de trabajo es elevada, la elasticidad de la oferta de trabajo es baja, la valoración de los parafiscales por parte de los trabajadores es pequeña y el poder de negociación de los trabajadores es exiguo.

\section{3. ¿Cómo estimar los efectos sobre el empleo de una rebaja de impuestos?}

Si el efecto de una reducción del impuesto al trabajo no es predecible a priori por la teoría económica, el proceso empírico de estimación se vuelve crucial para derivar indicaciones de política.

De hecho, como se deduce del análisis teórico y como bien resaltan Bennmarker et al. (2008: 3), "mientras la racionalidad de una reducción de los gravámenes a la nómina es clara y va en la dirección de incrementar el empleo, los resultados generalmente son inciertos. Mientras que el efecto de una disminución de estos impuestos sobre la demanda de trabajo es sin ambigüedades no negativo, es también posible que se produzcan contrastantes respuestas de oferta y aumentos de los salarios los cuales potencialmente dejan el empleo sin afectación".

Varios puntos deben ser aclarados para acercarse a una correcta estimación del impacto sobre el empleo de una reducción de los impuestos al trabajo.

Antes que nada, hay que reconocer que el impacto sobre el empleo de los impuestos a la nómina puede tener características asimétricas, que en caso de reducirse producirían un incremento del empleo muy inferior a las pérdidas ocupacionales causadas por un igual aumento de los mismos impuestos (Kugler y Kugler, 2008) ${ }^{10}$. Según Kesselman (1996: 173), esta asimetría se origina en el hecho de que "hay menores resistencias a subir salarios (en respuesta a menores impuestos) que a recortarlos (cuando los impuestos suben)". Asimismo, en el caso de la existencia de un salario mínimo, la ley no permite a las empresas reducir las remuneraciones de los trabajadores contratados con este salario si los impuestos a la nómina aumentan -i.e. el efecto pass through es igual a $0 \%-$, pero si los impuestos bajan ninguna disposición legal les impide reconocer salarios más altos, en el cual caso el efecto pass through podría ser distinto de $0 \%$. En especial, en presencia de negociación colectiva, empresarios y sindicatos pueden percibir la disminución de los gravámenes a la nómina como una "ganancia caída del cielo" que se reparten

\footnotetext{
${ }^{10}$ Más precisamente los autores sostienen que "puede haber un desplazamiento completo (del impuesto hacia los salarios) en respuesta de una importante reducción de los impuestos a la nómina y ningún o un desplazamiento incompleto en respuesta a un importante aumento" (Kugler y Kugler, 2008: 5). Lo anterior implica que, si aumentar la parafiscalidad reduce el empleo, su disminución no llevaría a mayores ganancias ocupacionales. De manera similar, en el caso de Argentina, Cruces, Galiani y Kidyba (2010) determinan la presencia de un efecto desplazamiento solo cuando los impuestos son reducidos.
} 
según el respectivo poder de negociación entre beneficios y salarios, sin mayores consecuencias sobre el empleo (Bennmarker et al., 2008).

De todo lo anterior se deriva una primera e importante observación: estimaciones del efecto sobre el empleo de un aumento de los impuestos a la nómina no pueden ser tomadas como referentes si, al contrario, se quiere conocer el impacto producido por una disminución de los mismos impuestos.

En el pasado, los efectos de variaciones en los gravámenes a la nómina eran generalmente derivados aplicando los valores conocidos de la elasticidad empleo-costos laborales. Este procedimiento, sin embargo, sufre de una grave limitación: no capta por completo el efecto desplazamiento del impuesto hacia los salarios; lo cual no solo no se concilia con el análisis teórico desarrollado en la sección 2 , sino que va en contra de una extensa literatura empírica internacional.

En países desarrollados, las investigaciones empíricas han encontrado porcentajes de "traslado" (pass through) elevados (Ooghe et al., 2003; Azemar y Desbordes, 2010), en algunos casos cercanos al $100 \%$, con lo cual el desplazamiento del impuesto hacia los salarios es prácticamente completo y los efectos sobre los niveles de empleo son marginales. Al inspeccionar las experiencias de algunos países desarrollados y en desarrollo, Taymaz (2006) concluye que, tanto en los primeros como en los segundos, gran parte de los impuestos a la nómina son trasladados a los salarios de los trabajadores y que el efecto pass through sería superior al 50\%. Este efecto, además, operaría en ambas direcciones, cuando los impuestos aumentan así como cuando disminuyen. Según el Banco Mundial, una revisión para
América Latina indicaría que "entre un 20 y un $70 \%$ del monto de las contribuciones a la seguridad social a cargo de los empleadores es trasladado a sus trabajadores" (World Bank, 2009: 16). Valores más altos han sido hallados por Gruber (1997), que en el caso de Chile obtiene un desplazamiento casi completo, y Cruces, Galiani y Kidyba (2010), que calculan un efecto pass through de entre 40 y $90 \%$ en Argentina. Por fuera de América Latina, en un país en desarrollo como Paquistán, Fatima (2012) también determina la existencia de un desplazamiento casi completo.

En resumen, en concordancia con lo que predice la teoría, en la práctica las empresas consiguen trasladar un porcentaje importante de los gravámenes a la nómina a sus propios empleados. Y viceversa, en el caso en que los impuestos bajen, los trabajadores logran apoderarse de una parte significativa de los alivios tributarios reconocidos a las empresas.

Más aun cuando, como en el caso de la Ley 1607 de 2012, la disminución de las contribuciones parafiscales es financiada por un aumento de los impuestos a los ingresos laborales de las personas naturales. Como consecuencia del mayor impuesto a la renta, la oferta de trabajo puede contraerse y la actitud negociadora de los trabajadores puede radicalizarse en pos de recuperar parte de él. Bajo estas circunstancias, es de esperar un aumento de los salarios y luego un menor efecto sobre el empleo.

Así que una correcta estimación del impacto ocupacional de una reducción de los impuestos al trabajo no puede basarse en el uso exclusivo de las elasticidades empleocostos laborales, dado que ellas "no capturan en su totalidad el efecto sobre el empleo", para lo cual hay que "tomar en considera- 
ción lo que los economistas llaman el efecto pass through -es decir, la proporción del impuesto que es endosado a los trabajadores" (World Bank, 2009: 16).

Podemos por lo tanto concluir que si se quiere comprobar las consecuencias ocupacionales de una reducción de los impuestos al factor trabajo, de poco sirven como guía los resultados de estimaciones que miden la relación causal opuesta-cuánto disminuye el empleo si aumenta el impuesto-y que no toman en consideración la existencia del efecto "traslado". Lo más apropiado es recurrir a una estimación directa sobre el empleo, explotando la existencia de disminuciones en el tiempo, o de diferencias entre áreas geográficas, en las tasas de impuesto a la nómina.

\section{4. ¿Cuando los impuestos al trabajo ba- jan, el empleo aumenta? Una revisión de los resultados en el ámbito internacional}

En la historia económica de Colombia son contados los episodios de rebajas de los impuestos al trabajo. Las leyes 50 de 1990 y 789 de 2002, de Reforma Laboral, disminuyeron los costos de contratar personal asalariado, pero al poco tiempo de su implementación estas disminuciones fueron compensadas por aumentos de las contribuciones a la seguridad social.

En diciembre de 2010, el actual gobierno tramitó la Ley 1429, de Formalización y Generación de Empleo, la cual ofrece a las empresas beneficios similares a los que otorga la Ley 1607 de 2012. Como consecuencia de las rebajas tributarias ofrecidas por la Ley 1429, se esperaba crear 350.000 empleos formales en cuatro años a consecuencia exclusiva de su aplicación. A diciembre del 2011, las 290.703 empresas beneficiarias solo afiliaron a seguridad social a 35.952 nuevos trabajadores (Ministerio del Trabajo de Colombia, 2012), menos de la mitad de los 87.500 empleos que debían generarse durante el primer año. Muchos de estos trabajadores, además, no le deben su empleo a la ley de Formalización, sino al acelerado crecimiento experimentado por la economía nacional y a los programas de formalización que en todo el país vienen desarrollando las Cámaras de Comercio, entre otros.

Si bien el gobierno nacional acaba de contratar un estudio de evaluación de impacto de la Ley 1429, actualmente en Colombia no existen trabajos empíricos que permitan derivar conclusiones confiables acerca de los probables efectos ocupacionales de la reducción de impuestos a la nómina prevista por la Ley 1607. Por tal razón, en esta sección recurrimos a una revisión de algunas experiencias internacionales.

En efecto, a partir de los años ochenta un creciente número de países del mundo ha venido reduciendo la presión fiscal sobre la nómina de sus empresas con la intención de estimular la generación de empleo. A pesar de las intenciones, la evidencia disponible tiende a mostrar ganancias ocupacionales marginales o nulas. A continuación se detallan los trabajos empíricos que sustentan esta afirmación ${ }^{11}$.

${ }^{11}$ Con respecto a las técnicas de estimación, vale la pena resaltar que, con excepción de los casos de la Unión Europea y del Perú, en todos los países considerados las estimaciones se basaron en la comparación de los resultados ocupacionales obtenidos en empresas de características similares, pero no todas beneficiarias de los recortes tributarios o no todas beneficiarias en la misma proporción. 


\section{Unión Europea}

Un reciente estudio financiado por la $\mathrm{Co}-$ misión Europea analiza los efectos sobre el mercado de trabajo de las reformas tributarias implementadas en un grupo de 31 países europeos, además de Japón y Estados Unidos, durante los años 1990-2008. Se revisaron 1.331 cambios al impuesto a la renta personal, 474 reformas a la seguridad social que modificaron las contribuciones a cargo de los trabajadores asalariados, 767 que modificaron aquellas de competencia de los empleadores y 417 que afectaron a los trabajadores autónomos. El análisis empírico se concentró solo en las reformas que redujeron la tributación a la renta personal y a la seguridad social, y verificó sus efectos sobre el número de horas laboradas semanalmente y las tasas de inactividad, desempleo y ocupación. El informe resalta cómo "en general (incluido el efecto de las reformas a la seguridad social sobre el empleo), el impacto de las reformas es cuantitativamente muy limitado y estadísticamente no significativo" (Econopubblica, 2011: 105). Además, avanza las siguientes recomendaciones de política: “a) la política tributaria puede jugar solo un rol limitado en la determinación de los resultados del mercado laboral (ocupación, desempleo, inactividad y horas trabajadas) comparada con medidas de política más efectivas como la negociación colectiva, las transferencias monetarias y en especie, los servicio de intermediación laboral, los programas de formación, las ayudas a la movilidad geográfica... b) en todo caso las reformas tributarias deberían siempre ser cuidadosamente coordinadas con otras herramientas de política... c) con el fin de registrar algún impacto sobre el mercado de trabajo, las políticas tributarias no deberían tener un carácter general, sino que deberían ser focalizadas hacia grupos particulares de trabajadores (mujeres casadas, madres solteras, trabajadores poco calificados)" (Econopubblica, 2011: 246).

\section{Argentina}

A partir de 1995, en Argentina se pasó de un sistema prácticamente uniforme de impuestos a la nómina a nivel territorial a uno diferenciado por área geográfica. Ese proceso fue acompañado por progresivas reducciones de los mencionados impuestos entre 1995 y 1999 y por un alza a partir de mediados de 2001, la cual, sin embargo, no alcanzó a restaurar nuevamente los niveles impositivos iniciales. Cruces, Galiani y Kidyba (2010: 19) estiman los efectos de estos cambios regionalmente diferenciados sobre los salarios y los asalariados formales del sector privado urbano y no encuentran "ningún efecto estadísticamente significativo sobre el empleo". Concluyen que "el principal argumento que justificó las reducciones del impuesto en 1995-1999 (los menores costos laborales estimularían una mayor ocupación) no fue confirmado por los hechos" (ídem: 20).

\section{Turquía}

Con el fin de estimular la inversión y la generación de empleo en las provincias de menores ingresos, el gobierno turco aprobó en 1998 una serie de incentivos en favor de las empresas residentes, los cuales incluían, entre otros, reducciones en las contribuciones patronales a la seguridad social. La cobertura geográfica de estos beneficios fue ampliada a un mayor número de provincias en 2004 y 2005. El Banco Mundial ha llevado a cabo una serie de ejercicios para determinar el impacto ocupacional y fiscal 
de una eventual reforma tributaria en Turquía, basándose en parte en la evaluación empírica de los mencionados programas de incentivos provinciales. Las conclusiones no fueron muy halagadoras y el informe de síntesis reconoce que "nuestra investigación sugiere que un impacto ocupacional de importancia puede ser alcanzado solo con una gran reducción de los impuestos al trabajo" (World Bank, 2009: 40). Como recomendación de política, el Banco Mundial aconseja evitar una reducción generalizada de los impuestos al trabajo ya que ello constituiría "un instrumento particularmente desfavorable para incrementar el empleo formal desde una perspectiva costo-beneficio" (ídem). Mejores resultados se obtendrían focalizando los recortes tributarios hacia los trabajadores de menores ingresos y/o jóvenes, para los cuales el efecto pass through es menor.

\section{Suecia}

Bennmarker et al. (2008: 29) estudian los efectos sobre la masa salarial y el empleo de la disminución de la tarifa de impuesto a la nómina aprobada en 2002, la cual favoreció a las pequeñas empresas del sector privado localizadas en algunas municipalidades del norte de Suecia, alejadas y escasamente pobladas. Comparándolas con un grupo de empresas de control con características similares localizadas en áreas cercanas, los autores "no encuentran que los diferenciales regionales de impuestos hayan tenido algún efecto significativo sobre el empleo" de las empresas.

\section{Finlandia}

A partir de enero de 2003, el gobierno finlandés decidió reducir, de forma temporal por tres años, los aportes a pensiones y salud de las firmas ubicadas en 20 municipalidades norteñas caracterizadas por altos niveles de desempleo. En años sucesivos estos beneficios fueron renovados en dos ocasiones hasta 2012 y extendidos a otras municipalidades del este del país.

Korkeamaki y Uusitalo (2006: 26) evalúan los efectos sobre el empleo y los salarios por hora de los primeros dos años de aplicación de estas medidas. Para ello definen un grupo de empresas de control ubicadas en municipalidades no elegibles para las reducciones y con características demográfico-laborales similares a las 20 municipalidades beneficiarias. Como resultado de la comparación entre los dos grupos de empresas, encuentran que en las regiones beneficiarias aproximadamente la mitad de la disminución de las contribuciones a seguridad social fueron trasladadas a mayores salarios. La restante disminución en el costo del trabajo "no tuvo efectos significativos sobre el empleo".

En un ejercicio similar, Korkeamaki (2011: 31) extiende el periodo de evaluación hasta el año 2006. En este caso también encuentra que "los recortes del impuesto a la nómina no tuvieron efectos estadísticamente significativos sobre el empleo total" de las empresas ubicadas en las áreas geográficas beneficiarias.

\section{Chile}

Gruber (1997) examina los efectos sobre los salarios y los niveles de empleo de la reducción en los gravámenes a la nómina que benefició a las firmas chilenas a principios de los años ochenta. Lleva a cabo estimaciones para un panel de empresas formales del sector manufacturero y encuentra coeficientes sobre el empleo pequeños y estadísticamente no significativos, acompañados por 
efectos "traslados" prácticamente completos, concluyendo, así, que "la menor carga impositiva parece haber sido pasada completamente a los trabajadores bajo la forma de salarios más altos, con un pequeño impacto sobre los niveles de empleo" (ídem: 99).

\section{Perú}

En 2003 entró en vigencia la Ley 28015, de Promoción y Formalización de la Micro y Pequeña Empresa, cuyo propósito era promover la competitividad, formalización y desarrollo de las micro y pequeñas empresas peruanas. Dicha ley y el sucesivo Decreto Legislativo 1086 de 2008 dieron vida a un régimen laboral especial para las empresas de hasta 10 trabajadores -microempresas$\mathrm{y}$ un régimen laboral especial intermedio para las empresas de hasta 100 trabajadores -pequeñas empresas. Inicialmente los dos regímenes tenían un carácter temporal que en 2008 se transformó en permanente. En el caso de las microempresas, el nuevo régimen laboral implicó un recorte de los costos laborales extrasalariales de más de 50 puntos porcentuales ${ }^{12}$. A pesar de la notable baja, los resultados obtenidos estuvieron muy por debajo de los pronosticados. El mismo Ministerio de Trabajo reconoció que "no se puede señalar que el régimen laboral especial creado por la Ley 28015 ha sido un régimen efectivo, dado que en 5 años y dos meses, registró a un total de 48.162 empresas y 84.261 trabajadores" (Ministerio de Trabajo de Perú, 2009: 21).

\section{5. ¿Cómo se ha proyectado el millón de nuevos empleos?}

Volviendo a Colombia, con la aprobación de la reforma tributaria en diciembre de 2012, el gobierno nacional estima generar un mínimo de 400 mil nuevos empleos. ¿Cómo se ha llegado a establecer ganancias ocupacionales tan cuantiosas comparadas con la experiencia internacional?

Antes de entrar en los detalles de la fórmula de cálculo, vale la pena resaltar que si bien todas las empresas -con ánimo de lucro-del sector privado se benefician de la reducción de 13,5 puntos porcentuales de parafiscalidad, para algunas de ellas -las intensivas en capital y de elevadas utilidades- la introducción del impuesto para la equidad provoca un aumento de los impuestos totales -suma de renta y parafiscalidad-asociados con el nivel de personal contratado en el momento de la entrada en vigencia de la Ley 1607. Según cálculos del Ministerio de Hacienda, los sectores económicos afectados por esta mayor carga impositiva son el financiero, el minero y de electricidad, gas y agua. Para las empresas de estos sectores, la reforma

\footnotetext{
${ }^{12}$ Con respecto al régimen laboral general, los trabajadores de las microempresas pierden el derecho al sobrecargo por trabajo nocturno -si éste es habitual-, a dos sueldos anuales por concepto de gratificaciones, a la compensación por terminación de servicio, a las asignaciones familiares por hijos dependientes y a un seguro de vida. Las vacaciones se reducen a la mitad y la indemnización por despido arbitrario, a un tercio. Además, las microempresas están exentas de pagar el seguro complementario de trabajo de riesgo y la contribución al Servicio Nacional de Adiestramiento en Trabajo Industrial. Finalmente, el empleador puede optar por afiliarse y afiliar a sus trabajadores al régimen contributivo de salud -EsSalud- o a un régimen semisubsidiado por el Estado -sis(ver, por ejemplo Sánchez, 2012).
} 
tributaria genera muy pocos incentivos para vincular laboralmente más trabajadores ${ }^{13}$. En situación similar se encuentran numerosos restaurantes y bares formales cuya estructura de costos lleva a que otras disposiciones previstas en la Ley 1607, como la sustitución del IVA -tarifas descontables de 5\% y $16 \%$ sobre las ventas- con un impuesto al consumo -tarifa no descontable de $8 \%$ sobre las ventas-y el incremento de la tarifa IVA sobre arriendos -de $10 \%$ a $16 \%$-, reduzcan sus utilidades comerciales. Los hoteles formales ofrecen servicio de restaurante en sus instalaciones, $\mathrm{y}$ adicionalmente han sido afectados por el aumento de la tarifa IVA sobre alojamiento de $10 \%$ a $16 \%$. A fin de cuentas, es sobre todo entre las pequeñas empresas agrícolas e industriales que se espera que la reforma tributaria estimule mayores contrataciones.

El Cuadro 1 clasifica los ocupados del año 2012 según ramas de actividad favorecidas o afectadas tributariamente por los cambios introducidos por la Ley 1607. Los datos discriminan entre asalariados formales y ocupados totales. Si a los valores agregados nacionales se les restan los ocupados en el sector público, en el sector educativo -que se toma como proxy del empleo en las entidades sin ánimo de lucro-, y de las ramas de intermediación financiera, minería y de suministro de electricidad, gas y agua, se llega a la conclusión de que, antes de la vigencia de la Ley 1607, en Colombia había algo más de cuatro millones de trabajadores asalariados en los sectores tributariamente favorecidos por la misma.

Así que, según el gobierno nacional, a los 4.177.432 de empleos asalariados ya existentes la rebaja tributaria ofrecida a la mayoría de las empresas formales del sector privado permitiría añadir entre 400 mil y un millón de empleos adicionales.

¿Cómo se estableció este incremento? En la exposición de motivos solo se señala que el número de nuevos puestos de trabajo se determinó de acuerdo con los cálculos realizados por tres estudios anteriores, ninguno de los cuales, desafortunadamente, cumple con los requisitos planteados en la sección 3 como necesarios para evaluar el auténtico efecto ocupacional de una reducción de los impuestos al trabajo.

Adicionalmente, en una reseña de varias investigaciones sobre demanda de trabajo en Colombia, el Ministerio de Hacienda estableció que la elasticidad empleo-costos laborales era del orden de entre $0,3 \%$ y $0,5 \%$, es decir que una reducción de $8 \%$ en los costos laborales induciría un aumento del empleo entre 2,4\% y 4\% (Cárdenas, 2012). El Cuadro 2 aplica estos porcentajes a los stocks de ocupados del Cuadro $1^{14}$. Naturalmente, se trata de un simple ejercicio contable que no pretende medir el efecto definitivo de la Ley 1607 sobre el empleo. Sin embargo, puede considerarse indicativo de los resultados es-

\footnotetext{
${ }^{13}$ Cabe notar que la reforma tributaria trae otro efecto sobre el empleo de las empresas afectadas: cambia el precio relativo entre el trabajo de la población de menores y mayores ingresos -capacitación-. Dada la tecnología prevaleciente en estos tres sectores -se trata de actividades intensivas en el uso de capital- es de suponer que la elasticidad de sustitución entre los dos tipos de trabajadores sea muy baja ya que para las empresas no sería costo efectivo cambiar la composición óptima de sus trabajadores.

${ }^{14}$ Este ejercicio se inspira en World Bank (2009), donde el efecto "traslado" se supone igual a $70 \%$.
} 


\section{Cuadro 1}

Ocupados según rama de actividad y categoría ocupacional. Total Nacional.

Promedio anual 2012

\begin{tabular}{|l|c|c|}
\hline \multicolumn{1}{|c|}{ Rama de actividad } & $\begin{array}{c}\text { Asalariados } \\
\text { formales }\end{array}$ & $\begin{array}{c}\text { Ocupados } \\
\text { totales }\end{array}$ \\
\hline Empleo total & 5.618 .057 & 20.696418 \\
\hline Empleo en los sectores para los cuales no aplica la reforma tributaria & & 837.531 \\
\hline Sector público & 837.531 & 473.875 \\
\hline Entidades sin ánimo de lucro (educación) & 240.706 & 249.951 \\
\hline Empleo en los sectores perjudicados por la reforma tributaria & & 234.702 \\
\hline Intermediación financiera & 194.838 & 89.582 \\
\hline Explotación de minas y canteras & 95.918 & 18.810 .777 \\
\hline Suministro de electricidad, gas y agua & 71.633 & \\
\hline Empleo en los sectores favorecidos por la reforma tributaria & 4.177 .432 & \\
\hline
\end{tabular}

Son formales los trabajadores que declaran estar afiliados al sistema pensional y los que ya están pensionados.

Fuente: DANE, Encuestas de Hogares.

perados a falta de verdaderas estimaciones de impacto ${ }^{15,16}$.

Los resultados obtenidos concuerdan con los reportados en la exposición de motivos por el gobierno nacional exclusivamente en el caso de aplicar el rango de elasticidades a la ocupación nacional total. Esta operación, sin embargo, implicaría que una disminución de los costos laborales en las empresas del sector formal privado provocaría un aumento no solo de su personal, sino también un proporcional crecimiento de los trabajadores independientes y de los trabajadores asalariados informales para los cuales la parafiscalidad no se modifica: dado que las elasticidades empleo-costos laborales se calculan para los asalariados, sería inadecuado emplearlas sobre la población ocupada total.

Así que las elasticidades empleo-costos laborales deben aplicarse solo a los asalariados formales. En estas circunstancias, las ganancias ocupacionales son notablemente inferiores y se ubican entre los $100.000 \mathrm{y}$ los 170.000 nuevos puestos de trabajo. Estas cifras, sin embargo, todavía no tienen en cuenta el traslado de parte del menor impuesto a los salarios. Asumiendo un valor conservador de 50\% para el efecto pass through, las ganancias ocupacionales se reducen después a 50.000-85.000 plazas. Un

\footnotetext{
${ }^{15}$ En especial, puede en alguna medida subestimar el efecto definitivo, por tratarse de un ejercicio a producción constante.

${ }^{16}$ En este contexto vale la pena recordar que si por un lado el costo de contratar mano de obra remunerada menos de diez salarios mínimos mensuales baja en un $8 \%$, por el otro las empresas terminan pagando más impuesto de renta. Esto se debe a que ya no son descontables como costos los aportes a SENA, ICBF y salud y a que la base de liquidación del nuevo impuesto para la equidad permite menos deducciones.
} 
Cuadro 2

Impacto sobre el empleo de la disminución de 13,5 puntos porcentuales de parafiscalidad

\begin{tabular}{|l|c|c|}
\hline \multicolumn{1}{|c|}{ Impacto respecto a: } & $\begin{array}{c}\text { Límite inferior } \\
\text { (elasticidad 0,3\%) }\end{array}$ & $\begin{array}{c}\text { Límite superior } \\
\text { (elasticidad 0,5\%) }\end{array}$ \\
\hline Ocupados totales & 496.714 & 827.857 \\
\hline Asalariados formales & 100.258 & 167.097 \\
\hline Asalariados formales con efecto pass through & 50.129 & 83.549 \\
\hline
\end{tabular}

Se asumen elasticidades empleo-costos laborales de 0,3\% y 0,5\% y un efecto pass through de $50 \%$.

Fuente: Elaboraciones del Observatorio del Mercado de Trabajo y la Seguridad Social con base en las elasticidades reportadas en Cárdenas (2012) y en datos del Cuadro 1.

resultado bastante menor de lo previsto por el gobierno nacional pero coherente con la experiencia internacional reseñada antes, y en línea con las indicaciones de otros estudios sobre el impacto ocupacional de la eliminación de la parafiscalidad en Colombia. Por ejemplo, según el modelo de equilibrio general de Hernández (2011), la eliminación de la parafiscalidad -en todos los sectores económicos y reemplazada por otro tipo de impuesto- no tiene efectos sobre la tasa de desempleo y modifica solo en un punto porcentual la tasa de informalidad. Asimismo, la sustitución generalizada de la parafiscalidad por impuestos indirectos llevaría a un incremento de 157.000 empleos formales, según las simulaciones de Botero (2011).

\section{6. ¿Habrá mayor equidad distributiva?}

Si el objetivo de generar más empleos muy difícilmente podrá alcanzarse, ¿al menos podrá lograrse una mejor distribución del ingreso con la implementación de la reforma tributaria?
Sin dudas, los cambios introducidos por la Ley 1607 en materia de tributación a la renta individual aumentarán el recaudo y harán que las personas de ingresos laborales más altos paguen más impuestos. Además, por lo discutido en la sección anterior algunos individuos encontrarán trabajo gracias a la reforma y esto influirá de manera positiva sobre la distribución personal del ingreso.

Sin embargo, mientras que, por un lado, las personas pagarán más renta, por el otro, la tributación de las empresas disminuirá de forma importante ${ }^{17}$. Con lo cual se dará una primera redistribución de carácter funcional-entre propietarios y capitalistas, por un lado, y asalariados, por el otro- del ingreso profundamente regresiva. Ahora bien, hay indicios de que esta mayor concentración del ingreso nacional a favor del capital, frecuentemente ha llevado a una mayor desigualdad general del ingreso de los hogares (OIT, 2012).

Debe resultar claro, entonces, que la reducción de la desigualdad medida a través del índice de Gini -que pasaría de 57,3 a 55,4 según la exposición de motivos- hace

${ }^{17}$ Como se ha aclarado en la introducción, en la práctica la reforma tributaria reduce la tributación de muchas empresas en un monto máximo cercano a 13,5 puntos porcentuales de sus nóminas. 
referencia a las rentas personales todavía no afectadas por el empeoramiento de la distribución funcional del ingreso, y por tanto no representa el efecto definitivo de la reforma tributaria, el cual sobreestima ${ }^{18}$.

Asimismo, mientras se incrementará la carga tributaria para las rentas laborales, quedaron algunas estridentes inequidades. Es el caso, por ejemplo, de las rentas por pensiones que la reforma tributaria ha mantenido exentas hasta el monto de 1.000 unidades de valor tributario. Así, quien trabaja empieza a pagar impuestos desde ingresos de 3.460 .000 mensuales, mientras los que gozan de una pensión, solo a partir de 26.800.000 de pesos mensuales.

En fin, que la reforma tributaria consiga una distribución efectivamente más equitativa de la riqueza nacional queda por demostrarse.

\section{Lecciones de política}

El carácter estructural que se le quiso atribuir a la reforma tributaria hubiera requerido una amplia, ponderada e incluyente discusión con la academia y los diversos estamentos de la sociedad colombiana. Por el contrario, el proyecto de reforma tributaria fue "cocinado" y aprobado por el Congreso de la República en la vuelta de apenas dos meses, tiempo absolutamente insuficiente para llevar a cabo un exhaustivo y serio análisis técnico de todos los aspectos de la reforma. Las consecuencias de la prisa legislativa están a la vista.
Contrario a lo que sostienen los promotores de la reforma, nuestro análisis prevé que ella muy difícilmente va a lograr un significativo incremento del empleo formal y que una mejora de la distribución del ingreso está en entredicho.

En cuanto a la creación de empleo, el gobierno nacional está en mora de explicar cómo determinó el monto de puestos de trabajo que generaría la reforma. Este resulta ser muy alto y con un generoso margen de error, y en consecuencia, poco creíble: los nuevos empleos podrían ser 400 mil, o dos veces y medio más. Al parecer, las elevadas ganancias ocupacionales que reporta el gobierno nacional son el resultado de simulaciones de modelos teóricos calibrados de forma no del todo afinada y de supuestos demasiados optimistas acerca de la sensibilidad del empleo frente a disminuciones de los gravámenes a la nómina. Utilizando un rango de elasticidades empleo-costos laborales que el mismo gobierno nacional estableció con base en la reseña de varios estudios sobre demanda de trabajo en Colombia y un prudente porcentaje de traslado del rebajado impuesto a la nómina hacia mayores salarios, hemos replicado un simple ejercicio realizado en World Bank (2009) y encontrado que el número de nuevos empleos generados por la reforma no sería superior a los 85.000. Muy poco para justificar una reforma tributaria en Colombia.

El gobierno colombiano no solo desestimó los aportes empíricos de una abundante literatura internacional-que coincide en no

\footnotetext{
${ }^{18}$ Tampoco hay que excluir que los trabajadores más afectados por el mayor impuesto a la renta, dado su poder de negociación, puedan restaurar parcial o totalmente su capacidad de consumo a través de incrementos salariales o de la utilización de parte de las mayores ganancias empresariales.
} 
encontrar impactos significativos sobre el empleo como consecuencia de reducciones de los impuestos gravados al factor trabajosino que hizo caso omiso de unas importantes recomendaciones de política avanzadas por esta literatura.

Tanto en el informe sobre reformas tributarias de Econopubblica (2011) para la Comisión Europea como en el del Banco Mundial para Turquía se recomienda no proceder a una baja generalizada de la parafiscalidad. Más bien se sugiere reducir los impuestos al trabajo no calificado, de salario mínimo, o de colectivos específicos, como los jóvenes, las mujeres casadas o cabeza de hogar. Para estas categorías de trabajadores el efecto pass through es mucho más pequeño que para los demás trabajadores y, en consecuencia, es mayor el efecto sobre el empleo. Según lo aprobado con la reforma, al contrario, la rebaja tributaria termina aplicándose a más del $90 \%$ de los trabajadores contratados por empresas con ánimo de lucro. Casi todos.

Asimismo, la estrategia de creación de empleo presentada recientemente por la Comisión Europea a los Estados miembro aboga también por un desplazamiento de la carga fiscal del trabajo hacia otras fuentes, pero primordialmente hacia los impuestos medioambientales ${ }^{19}$.

En cuanto a la distribución del ingreso, la reforma exonera a las empresas del pago de aproximadamente 6,7 billones de pesos, correspondientes a los 13,5 puntos porcentuales de parafiscalidad que dejan de contribuir. La financiación de la salud de sus trabajadores, del SENA y del ICBF pasa a ser sufragada en su mayor parte por el aumentado impues- to a la renta de las personas naturales. Esta considerable reasignación de recursos tendrá consecuencias regresivas sobre la distribución personal del ingreso y puede comprometer la esperada consecución de una mejor equidad distributiva derivable de una tabla de impuesto a la renta más progresiva.

Tampoco la reforma aprobada hace parte de una estrategia integral de lucha contra la informalidad. Una estrategia de este tipo podría incluir, por ejemplo, la coordinación de los servicios ofrecidos por el Estado y la divulgación de los beneficios de la formalización, el diálogo con sectores de la economía con mayores índices de informalidad, la introducción de un sistema tributario sencillo -para una población específica- y que promueva la visibilidad de las actividades económicas del país, acciones en el campo de la educación e inclusión financiera, y una mayor fiscalización por parte del Estado. De hecho, estas son las propuestas contenidas en un documento del Centro de Estudios Fiscales del Ministerio de Hacienda, elaborado apenas un año antes de la presentación al Congreso Nacional del proyecto de reforma tributaria y que, por extraño que pueda parecer, no propone reducción alguna de los impuestos al trabajo ${ }^{20}$. ¿En qué momento, en el Ministerio de Hacienda se descubrió la trascendental importancia de los 13,5 puntos porcentuales de parafiscalidad?

En fin, es probable que el sistema tributario colombiano, y en especial los impuestos que gravan la nómina de trabajadores, hubiese necesitado ser reformado. Sin embargo, la recién aprobada reforma tributaria ha sido un ejemplo de implementación de política me-

\footnotetext{
${ }^{19}$ Y también hacia impuestos al consumo y a la propiedad (European Commission, 2012).

${ }^{20}$ Ver Bustamante (2011).
} 
jorable bajo muchos aspectos. En especial, habría sido importante que hubiera tenido en mayor consideración:

- Las experiencias y las sugerencias de política de la más reciente literatura económica especializada que, entre otras, previene de aplicar una reducción generalizada de los impuestos al trabajo. Lo anterior tiene como corolario que el impacto previsto por el gobierno en materia de empleo y distribución del ingreso no cuenta con el debido sustento empírico.

- La posibilidad de implementar una estrategia integrada de lucha contra la informalidad, en vez de limitarse a operar exclusivamente sobre los costos no salariales - La relación con la política salarial. Como se ha comentado en la sección 2, parte del éxito en términos de empleo de una reducción en la tributación al trabajo depende del control que ejerce el Estado sobre la evolución del salario mínimo. En Colombia, éste aumentó $2,2 \%$ en términos reales el año pasado; en el mismo año la inflación se ubicó en $2,4 \%$. A pesar de lo anterior, el incremento fijado por el gobierno para 2013 fue de 4\%. Es evidente que para fines de generación de empleo las alzas en el salario mínimo concedidas por el gobierno compensan -en parte- la reducción en la parafiscalidad que el mismo gobierno propició con la reforma tributaria. En especial, ceteris paribus, por cada punto porcentual en que aumenta el salario mínimo se requiere de una disminución de 1,43 puntos porcentuales en la parafiscalidad para mantener inalterado el costo total de la nómina.

- La necesidad de dedicar un mayor tiempo a su elaboración y una mayor disposición a escuchar las opiniones y propuestas de otros estamentos de la sociedad colombiana. Así lo consideró, por ejemplo, la Contraloría General de la República (2012: 10) que en su momento afirmó que "no sería prudente ni conveniente que se tramitara el proyecto en lo que resta de la presente legislatura, sino más bien que se aplazara su tramitación a la próxima legislatura para dar la oportunidad de tener un debate más amplio en círculos académicos, en el gobierno y en el mismo Congreso de la República, y, por supuesto, en la opinión pública, como condición indispensable para acertar con una reforma tributaria que aporte en el proceso de construcción de una sociedad más democrática e incluyente".

\section{Bibliografía}

Azemar, C. y Desbordes, R. (2010). "Who Ultimately Bears the Burden of Greater Non-Wage Labour Costs?", en University of Strathclyde, Discussion Papers in Economics n. ${ }^{\circ}$ 10-04, Glasgow.

Borjas, G. (2005). Labor Economics. McGraw-Hill, 3 ed.

Botero, J. (2011). Desempleo e Informalidad en Colombia. Un Modelo de Equilibrio General Computable. mimeo, Universidad EAFIT, 9 de octubre.

Bustamante J.P. (2011). "Los Retos de la Economía Informal en Colombia”, en Notas Fiscales n. ․ 9. Bogotá: Ministerio de Hacienda y Crédito Público, Centro de Estudios Fiscales, agosto.

Cárdenas, M. (2012). Reforma Tributaria 2012. presentación ppt, 26 de octubre. 
Contraloría General de la República (2012). Comentario de la Contraloría General de la República al Proyecto de Ley No 166/2012 C, 134/12 S, por la Cual se Expiden Normas en Materia Tributaria y se Dictan Otras Disposiciones. 30 de noviembre.

Cruces, G.; Galiani, S., y Kidyba, S. (2010). "Payroll Taxes, Wages and Employment: Identification through Policy Changes", en Labour Economics, Vol. 14, n. ${ }^{\circ}$.

Econopubblica (2011). “The Role and Impact of Labour Taxation Policies”, en Informe para la Comisión Europea. Milan, Italia: Universitá Bocconi, may.

European Commission (2012). "Towards a Job-rich Recovery", en Communication from the Commission to the European Parliament, the Council, the European Economic and Social Committee and the Committee of the Regions. Strasbourg, 18 de abril.

Gamero, J. y Carrasco, G. (2011). Trabajo Informal y Políticas de Protección Social. Lima, Perú: WIEGO-CIES.

Gruber, J. (1997). “The Incidence of Payroll Taxation: Evidence from Chile”, en Journal of labor Economics, Vol. $15,{ }^{\circ}{ }^{\circ} 3$.

Hernández, G. (2011). "Impuestos Parafiscales y Mercado Laboral: un Análisis de Equilibrio General Computable", en Archivos de Economía, Documento n. ${ }^{\circ}$ 378, Septiembre.

Kesselman, J. (1996). "Payroll Taxes in the Finance of Social Security”, en Canadian Public Policy, Vol. XXII, n. ${ }^{\circ} 2$.

Korkeamaki, O. (2011). “The Finnish Payroll Tax Cut Experiment Revisited”, en Government Institute for Economic Research, vATT Working Paper n. ${ }^{\circ} 22 / 2011$, april.

Korkeamaki, O. y Uusitalo, R. (2006). "Employment Effects of a Payroll-Tax Cut. Evidence from a Regional Tax Exemption Experiment", en IFAU. Working Paper, 2006: 10, October.

Kugler, A. y Kugler, M. (2008). "Labor Market Effects of Payroll Taxes in a Developing Countries. Evidence from Colombia", en NBER. Working Paper n. ${ }^{\circ}$ 13855, March.

Layard, R.; Nickell, S., y Jackman, R. (1991). Unemployment: Macroeconomic Performance and the Labour Market. Oxford University Press.

Melguizo, A. y González-Páramo, J. (2012). "Who Bears Labour Taxes and Social Contributions? A Meta-analysis Approach", en Journal of the Spanish Economic Association, november.

Ministerio del Trabajo de Colombia (2012). Informe de Actividades 2011-2012, al Honorable Congreso de la República. Bogotá, julio.

Ministerio de Trabajo de Perú (2009). Micro y Pequeñas Empresas: Régimen Laboral Especial de la Nueva Ley Mype. Mimeo, agosto.

Ooghe, E.; Schokkaert, E., y Flechet, J. (2003). "The Incidence of Social Security Contributions: an Empirical Analysis", en Empirica, Vol. 30, n. ${ }^{\circ}$.

Organización Internacional del Trabajo -OIT- (2012). Informe Mundial sobre Salarios 2012/13. Geneva, diciembre.

República de Colombia (2012). "Proyecto de Ley No 166 de 2012”, en Gaceta del Congreso, Año XXI, n. 666, 5 de octubre.

Sánchez, C. (2012). "Una visión ponderada de la legislación laboral: comentarios al Régimen MYPE y a la Propuesta de «Ley de la Nueva Empresa»", en Derecho PUCP, n. ${ }^{\circ}$ 68, Pontificia Universidad Católica del Perú. 
Summers, L. (1989). “What Can Economics Contribute to Social Policy?", en American Economic Review Papers and Proceedings, Vol. 79, . $^{\circ}$ 2, may.

Taymaz, E. (2006). "Labor Demand in Turkey", Informe para el Banco Mundial. Mimeo, august.

World Bank (2009). "Estimating the Impact of Labor Taxes on Employment and Balance of the Social Insurance Funds in Turquey", en Synthesis Report, Human Development Sector Unit, Europe and Central Asia Region, Report n. ${ }^{\circ} 44056-\mathrm{TR}$, apr. 